\title{
GARRAPATAS (IXODIDA) Y ÁCAROS (MESOSTIGMATA) PARÁSITOS DE ROEDORES SIGMODONTINOS DEL DELTA DEL PARANÁ, ARGENTINA
}

\author{
Colombo, v. C. ${ }^{1} ;$ Lareschi, M. ${ }^{2}$; Monje, L. D. ${ }^{1} ;$ Nava, S. ${ }^{3}$; \\ AntoniazZi, L. R. ${ }^{1}$; Beldomenico, P. M. ${ }^{1}$ \& Guglielmone, A. A. ${ }^{3}$
}

\begin{abstract}
RESUMEN
Se determinaron las especies de garrapatas y ácaros mesostigmata parásitos de roedores sigmodontinos en una localidad del Delta del Paraná. Fueron registradas 38 asociaciones parásitohospedador entre dos especies de garrapatas y cinco especies de ácaros con seis especies de roedores, que mostraron una distribución agregada de sus parásitos. Dos de las asociaciones (Holochilus brasiliensis-Amblyomma triste y $H$. brasiliensis-Ixodes loricatus) son nóveles, siendo la asociación $H$. brasiliensis-Am. triste de importancia sanitaria ya que esta garrapata es vector de Rickettsia parkeri, patógeno que afecta a los humanos. La mayoría de las asociaciones se detectaron previamente sólo en la eco-región correspondiente a la localidad de estudio. Por el contrario, unas pocas asociaciones ya registraban antecedentes para otras regiones argentinas, el Brasil, Paraguay o el Uruguay.

Palabras clave: Garrapatas, ácaros, roedores, asociaciones, Paraná.
\end{abstract}

\section{SUMMARY}

Ticks (ixodida) and mites (mesostigmata) parasites of sigmodontine rodents in the Delta of Paraná river, Argentina.

The species of ticks (Ixodida) and mites (Mesostigmata) parasites of sigmodontine rodents were determined in a locality of the Paraná River Delta. A total of 38 host-parasite associations between two species of ticks and five species of mites were found from six species of rodents. Aggregated distributions of parasites on hosts characterized those associations. Two associations (Holochilus brasiliensis-Amblyomma triste y H. brasiliensis-Ixodes loricatus) were not registered before, and the H. brasiliensis-Am.triste association is of sanitary importance because this tick is a vector of

1.- Laboratorio de Ecología de Enfermedades, Instituto de Ciencias Veterinarias del Litoral, CONICETUNL. Kreder 2805. (3080) Esperanza, provincia de Santa Fe.

2.- Centro de Estudios Parasitológicos y de Vectores (CEPAVE), CCT-La Plata-UNLP, Calle 2

$\mathrm{N}^{\circ}$ 584. (1900) La Plata, provincia de Buenos Aires.

3.- Instituto Nacional de Tecnología Agropecuaria (INTA), Estación Experimental Agropecuaria Rafaela, INTA. CC 22. (2300) Rafaela, provincia de Santa Fe.

Manuscrito recibido el 8 de abril de 2013 y aceptado para su publicación el 16 de abril de 2013. 
rickettsiosis to humans. The majority of the associations were previously found only in the ecoregion where the study site is located. In contrast a few of the associations reported have antecedents in other regions of Argentina, Brazil, Paraguay or Uruguay.

Key words: Ticks, mites, rodents, associations, Paraná.

\section{INTRODUCCIÓN}

Los pequeños roedores se caracterizan por su amplia diversidad de parásitos (Krasnov et al., 2006), así el estudio de los ensambles entre ellos y sus parásitos son relevantes para conocer la biodiversidad de un ecosistema y sus aspectos epidemiológicos, lo cual se torna aún más importante cuando esas asociaciones incluyen organismos nocivos para los animales o el hombre. Varios investigadores estudiaron los diversos ectoparásitos de los roedores de la subfamilia Sigmodontinae (Rodentia, Cricetidae) en localidades de la porción inferior bonaerense del delta del río Paraná y del estuario del río de La Plata en la Argentina (Mauri, 1966; Mauri \& Capri, 1972; Lareschi, 1996, 2004, 2006, 2010 a; Lareschi \& Sánchez López, 2000; Liljesthröm \& Lareschi, 2002; Lareschi et al., 2003a; Nava et al., 2003; Navone et al., 2009; Lareschi \& Krasnov, 2010), mientras que Nava et al., (2004) y Beldomenico et al., (2005) investigaron específicamente el parasitismo de sigmodontinos con la garrapata Ixodes loricatus y Nava et al. (2011) estudiaron la ecología de Amblyomma triste, demostrando así la importancia de estos roedores para su ciclo. Trabajos similares en la Argentina incluyen una localidad en Entre Ríos (Abba et al., 2001), varias localidades del noroeste argentino (Lareschi et al., 2003 b), un sitio en Corrientes (Lareschi et al., 2006 a) y otro en Córdoba (Nava \& Lareschi, 2012). En tanto que el rol de los sigmodontinos como hospedadores de garrapatas en la Argentina y el resto del Neotrópico, fue revisado en Guglielmone \& Nava (2011) con información adicional para las garrapatas incluidas en esta investigación en Debárbora et al. (2012) y Ogrzewalska et al. (2012).

La información de esos estudios adquirieron mayor relevancia por el diagnóstico de rickettsiosis por Rickettsia parkeri en humanos transmitida por Am. triste en el delta del Paraná (Seijo et al., 2007; Nava et al., 2008; Romer et al., 2011), garrapata que fuera confundida previamente en la región con Amblyomma maculatum (Boero, 1955; Capri \& Mauri, 1971; Lareschi \& Sánchez López, 2000). En esta ocasión se presentan resultados generales, obtenidos durante el primer año de estudio, de las asociaciones de roedores sigmodontinos con garrapatas y ácaros mesostigmata para una localidad del delta del Paraná.

\section{MATERIALES Y MÉTODOS}

El trabajo de campo se realizó en la Estación Experimental Delta del Paraná del Instituto Nacional de Tecnología Agropecuaria (INTA Delta) $\left(34^{\circ} 11^{\prime} \mathrm{S}, 58^{\circ} 50^{\prime} \mathrm{W}\right)$, partido de Campana, provincia de Buenos Aires, Argentina, que corresponde a la provincia fitogeográfica Pampeana del Dominio del Chaco en Cabrera (1976) y a la eco-región Islas y Delta del Paraná en Burkart et al. (1999). Se capturaron roedores por tres días consecutivos cada cinco semanas en diferentes sitios del INTA Delta, desde septiembre de 2010 hasta agosto de 2011. En cada oca- 